\title{
Decoupled versus coupled growth dynamics of an irregular eutectic alloy
}

\author{
Samira Mohagheghi ${ }^{1}$, Sabine Bottin-Rousseau ${ }^{2}$, Silvère Akamatsu², Melis Şerefoğlu ${ }^{1 *}$ \\ ${ }^{1}$ Koç University, Department of Mechanical Engineering, Rumeli Feneri Yolu, 34450, Sariyer, Istanbul, \\ Turkey \\ ${ }^{2}$ Sorbonne Université, CNRS UMR 7588, Institut des NanoSciences de Paris, 4 Place Jussieu, 75252 Paris \\ Cedex 05, France
}

Keywords: Irregular eutectic growth; directional solidification; eutectic solidification; interface dynamics; solidification microstructures

\begin{abstract}
We present an experimental study of irregular growth patterns observed in real time during thin-sample directional solidification of a faceted/nonfaceted eutectic alloy, namely, the transparent 2-amino-2-methyl-1,3-propanediol (AMPD)-succinonitrile (SCN) system. The body-centered cubic SCN crystals are nonfaceted, while monoclinic AMPD crystals grow as faceted needles. At low velocities $\left(<0.3 \mu \mathrm{ms}^{-1}\right)$, a decoupled growth regime is observed, during which the tip of the AMPD crystals grows ahead of the SCN-liquid interface. At intermediate velocities, an unsteady coupled-growth regime takes place, with intermittent pinning of triple SCN-AMPD-liquid junctions, and frequent noncrystallographic branching. At higher velocities $\left(>1 \mu \mathrm{ms}^{-1}\right)$, two-phase fingers form.
\end{abstract}

Many irregular eutectic alloys of industrial importance are such that one of the solid phases is faceted (e.g., silicon, germanium, graphite), while the other one (e.g., aluminum, austenite) is nonfaceted [1-3]. In spite of extensive research, the growth of such faceted/nonfaceted (or $\mathrm{f} / \mathrm{nf}$ ) eutectics is still poorly understood. The term irregular refers to the disordered microstructures left frozen in the solid behind the growth front, whereas regular eutectics grow with fully nonfaceted solid-liquid interfaces. The growth dynamics of regular eutectics is determined by solute diffusion in the liquid, and local equilibrium at the solid-liquid interfaces, and at the triple-contact lines (trijunctions) between the liquid and the two solids [4]. During directional solidification (DS) at velocity $V$ in a temperature gradient $G$, steady periodic two-phased patterns can then form in a quasi-planar geometry at a temperature close to the eutectic point. The diffusive coupling between the growing entities (e.g., lamellae or rods) extends over distances much larger than the interphase spacing $\lambda$-hence the coupledgrowth appellation. In contrast, the unsteadiness of the growth dynamics of $\mathrm{f} / \mathrm{nf}$ eutectics is attributable to a nonlinear growth kinetics of the faceted interfaces. A facet can remain macroscopically immobile irrespective to the diffusion field over a finite range of undercoolings ("blocked" facet). The mobility of a facet is tributary of active sources of 
atomic or molecular step flow, such as emerging dislocations, the operation of which depends on geometrical features. The assumption of local equilibrium at interfaces is then no longer valid. Thus presented, the identification of basic, non-specific features of irregular eutectics seems, at the very least, a difficult problem. On the basis of real-time observations during thin-sample DS of transparent organic alloys, some conjectural models of irregular growth with a majority of nonfaceted phase in the solid have been proposed $[5,6]$. Two features were more or less explicitly postulated: the existence of stable trijunctions, which is questionable, and a frequent branching of the faceted phase, the noncrystallographic character of which was often omitted. The problem therefore eludes an analysis à la Jackson and Hunt. As emphasized in a recent study [7], irregular eutectic growth often characterizes by a transient "decoupling" between the growth of the faceted and the nonfaceted crystals. The question of the possibility of a steady-state $\mathrm{f} / \mathrm{nf}$ eutectic growth regime thus remains to be addressed properly.

In this Letter, we present an experimental study of irregular eutectic growth during thinsample DS of the 2-amino-2-methyl-1,3-propanediol (AMPD)-succinonitrile (SCN) eutectic alloy observed in real time. The alloy was slightly hypoeutectic (AMPD side). The SCN crystals are nonfaceted [8], whereas AMPD ones grow faceted from the melt [9]. We found three different two-phased growth regimes according to the pulling velocity: (i) a decoupled growth at low $V$, during which the leading end of needle-shaped AMPD crystals grows ahead of the quasi-planar SCN-liquid interface -in contrast to Ref. [7]; (ii) an unsteady coupledgrowth dynamics at intermediate velocities, with frequent noncrystallographic branching of AMPD crystals at, or near trijunctions; (iii) the formation of two-phase fingers at higher velocities.

Experimental procedure. The AMPD-SCN eutectic (temperature $\mathrm{T}_{\mathrm{E}}=325.7 \mathrm{~K}$; concentration $\mathrm{C}_{\mathrm{E}}=97.39 \mathrm{~mol} \% \mathrm{SCN}[9]$ ) involves the (quasi-pure) BCC SCN phase, and the monoclinic AMPD phase. The eutectic plateau is markedly asymmetric; the volume fraction of AMPD in the solid is of a few percents. Commercial SCN (AMPD) was purified by repeated distillation and degassing (sublimation). However, a morphological instability of the solidification front was observed for V higher than, say, $1 \mu \mathrm{ms}^{-1}$, due to residual impurities (the amount of which was slightly sample dependent). Alloys of slightly hypoeutectic concentration $C_{0}\left(\lesssim C_{E}\right)$ were prepared by melting and mixing the compounds under Argon atmosphere. Thin samples were made of two glass plates separated by $12-\mu \mathrm{m}$ thick spacers and glued to each other. The empty space (lateral dimensions: $6 \times 60 \mathrm{~mm}^{2}$ ) was filled with the molten eutectic alloy under an Argon atmosphere. Directional solidification was performed in a fixed temperature gradient 
$\left(7.5 \pm 0.5 \mathrm{Kmm}^{-1}\right)$ with $\mathrm{V}$ in the $0.01-2.00 \mu \mathrm{ms}^{-1}$ range. The growth front was observed in real time with an optical microscope, and a numerical camera.

Decoupled growth. At low velocities $\left(\mathrm{V}<0.3 \mu \mathrm{ms}^{-1}\right)$, a decoupled-growth regime was observed [Fig. 1(a)]. Thin AMPD crystals with a faceted, needle-like morphology were growing far apart from each other, with their leading tip ahead of the quasi-planar SCN-liquid interface. They were generally tilted from the temperature-gradient axis $\mathbf{z}$ (this was determined during the early stages of the solidification). Their lateral size hardly exceeded 20 $\mu \mathrm{m}$-very thin, optically hardly resolved AMPD crystals were also observed. They were delimited by blocked lateral facets that did not grow on a micron scale [10] except in rare, sudden events. Thin AMPD crystals could grow without contacting the sample walls over long times. When growing in opposite directions, some of them could even cross each other in the thickness of the sample without visible interactions and any reaction of the lateral facets. Let us define $1_{\mathrm{A}}$ as the protrusion length of an AMPD crystal (projected on $\mathbf{z}$ ) in the liquid, and $\Delta \mathrm{T}$ the difference of temperature between the tip and the $\mathrm{SCN}$-liquid interface $\left(\Delta \mathrm{T}=\mathrm{Gl}_{\mathrm{A}}\right)$. At $\mathrm{V}=0.01 \mu \mathrm{ms}^{-1}$, the longest AMPD crystals were such that $\mathrm{l}_{\mathrm{A}} \approx 250 \mu \mathrm{m}$, which corresponds to a maximum $\Delta \mathrm{T}$ value $\left(\Delta \mathrm{T}_{\max }\right)$ of about $2 \mathrm{~K}$. The $\mathrm{SCN}$-liquid interface remained planar on a large scale in the vicinity of AMPD crystals as long as $1_{\mathrm{A}}$ remained larger than, say, $100 \mu \mathrm{m}$ [Fig. 1(a)]. The SCN-liquid interface was observed to grow forward along shorter AMPD crystals $\left(1_{\mathrm{A}}<100 \mu \mathrm{m}\right)$. This gives a rough indication on the loose diffusive interplay between the tip of an AMPD crystal and the SCN crystal at that velocity.

In most cases, the SCN-liquid interface could be seen through thin transparent AMPD crystals, at fluctuating z positions [Fig. 1(a), inset 1]. Some AMPD crystals were thick enough to connect the two sample walls. In such cases, the SCN-liquid interface was contacting the two sides of the AMPD crystal at substantially different temperatures [Fig. 1(a), inset 2; also see Fig. 2(d) of Ref. [6]]. This configuration does not correspond to an equilibrated pinning of a trijunction: the SCN-liquid interface formed a meniscus near a blocked AMPD facet, like along a neutral surface -e.g., a glass plate. The above features -weak diffusive interplay, absence of equilibrated trijunctions- define a quasi-steady decoupled growth regime. They were observed reproducibly at low velocities. 

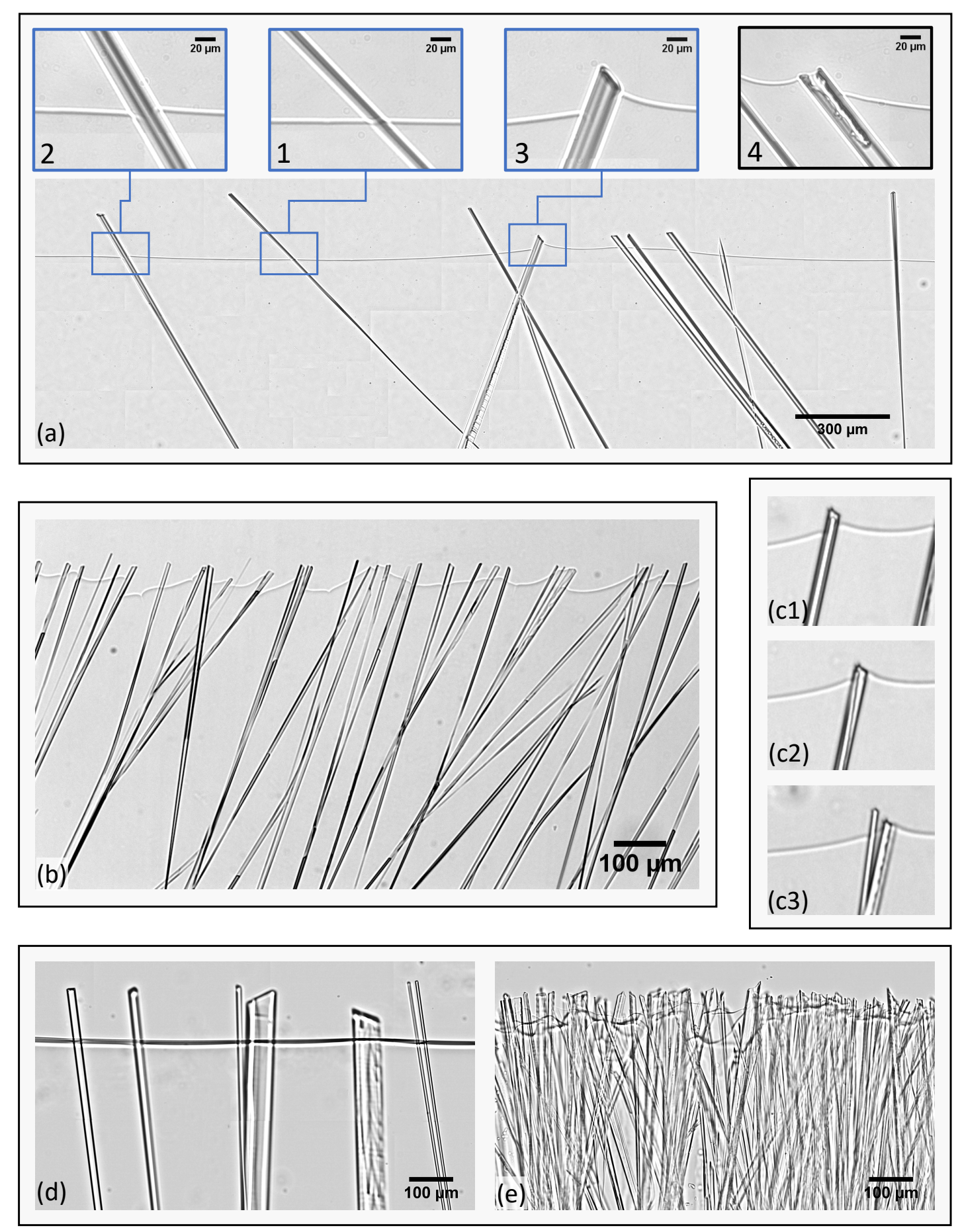

Fig. 1. Irregular growth patterns of the AMPD-SCN eutectic alloy during directional solidification in thin [(a) to (c)] and 100- $\mu \mathrm{m}$ thick [(d) and (e)] samples. (a) Decoupled growth at a low pulling velocity $\left(\mathrm{V}=0.05 \mu \mathrm{ms}^{-1}\right)$. Thin AMPD crystals grow tilted ahead of the SCN-liquid interface. Insets 1-3: large-magnification views of the framed details. Inset 4: same sample $\left(\mathrm{V}=0.10 \mu \mathrm{ms}^{-1}\right)$. (b) Unsteady coupled growth $\left(\mathrm{V}=0.60 \mu \mathrm{ms}^{-1}\right)$. c) Time sequence ( $\mathrm{V}=0.10 \mu \mathrm{ms}^{-1}$ ) showing: a decoupled pattern (c1), the pinning of a trijunction (c2), and noncrystallographic branching (c3). Horizontal dimension: $157 \mu \mathrm{m}$; time interval: $1340 \mathrm{~s}$. (d) Decoupled, and (e) unsteady coupled growth patterns in a thick sample $\left(\mathrm{V}=0.10\right.$ and $0.60 \mu \mathrm{ms}^{-1}$, respectively; $\left.\mathrm{G}=8.5 \mathrm{Kmm}^{-1}\right)$. The growth direction $\mathbf{z}$ is upward (the liquid on top) in all images. 
Let us put closer focus on individual AMPD crystals. The growth velocity of the tips was observed to vary in a non-monotonous way, and their shape (number and orientation of the facets) also changed over time. In rare occasions, the growth rate of a given AMPD crystal was observed to decrease so that the tip, while recoiling downwards in the temperature gradient, eventually reached the same level as the SCN-liquid interface. A pinning of the three (AMPD-liquid, SCN-liquid, and AMPD-SCN) interfaces could then occur at a $\mathbf{z}$ position, presumably close to $\mathrm{T}_{\mathrm{E}}$, located between the foremost AMPD tip and the SCN-liquid interface (far away from the structure in question). A solitary coupled-growth pattern with a regular trijunction thus formed [Fig. 1(a), inset 3], at least temporarily (and often on one side of the AMPD crystal only). The blocked AMPD facet was no longer exposed to the liquid. The trijunction was free to move laterally, and the AMPD crystal thickened. This dynamics went generally along with a substantial modification of the shape of the AMPD-liquid interface and the SCN-liquid interface. This evidences a local coupling -and the absence of a proper coupling otherwise. Addressing the question of the pinning angles of strongly anisotropic interfaces at the trijunction $[11,12]$ is beyond the scope of the present paper. In a coupledgrowth configuration [Fig. 1(a), inset 4], the AMPD crystals could adopt a complicated shape, reminiscent of the C-shaped crystals that were often reported in irregular eutectics [13, 14]. A splitting of the AMPD crystal could also occur, thus giving rise to the parallel growth of two thinner AMPD crystals with the same orientation (or with a very small misorientation of less than one degree, comparable to that of a subboundary). Noncrystallographic branching was not observed at low velocities. Localized coupled-growth shapes ended with a depinning of the trijunction, and a rapid growth of the AMPD crystal tip. The decoupled-growth morphology was then recovered.

The faceted nature of the leading tips of the AMPD crystals was therefore the main source of unsteadiness during the decoupled growth of the alloy under consideration. It is thus tempting to imagine that a steady-state decoupled-growth regime could establish in a $\mathrm{f} / \mathrm{nf}$ alloy with nonfaceted ends of the faceted crystals.

Unsteady coupled growth. Upon increasing $\mathrm{V}$, the difference of temperature between the AMPD tips and the SCN interface decreased (Fig. 2). This could be anticipated: the temperature of the AMPD tips with a kinetics-controlled growth was indeed expected to decrease when $\mathrm{V}$ increases. In contrast, the average temperature of the SCN-liquid interface 
did not depend on $\mathrm{V}$, but underwent large-scale deformations under the effect of a nonuniform diffusion field. The decrease of $\Delta \mathrm{T}_{\max }$, that is, in practice, the fact that the AMPD tips were getting closer to the SCN-liquid interface, played in favor of a more frequent pinning of AMPD-SCN-liquid trijunctions as V increased. Indeed, the formation of coupled-growth structures is one of the main features of the intermediate-velocity regime $(0.30<\mathrm{V}<0.60$ $\mu \mathrm{ms}^{-1}$ ) [Fig. 1(b)]. The growth dynamics was however clearly unsteady, with intermittent coupling and decoupling (i.e., pinning and depinning of the trijunctions) and noncrystallographic branching.

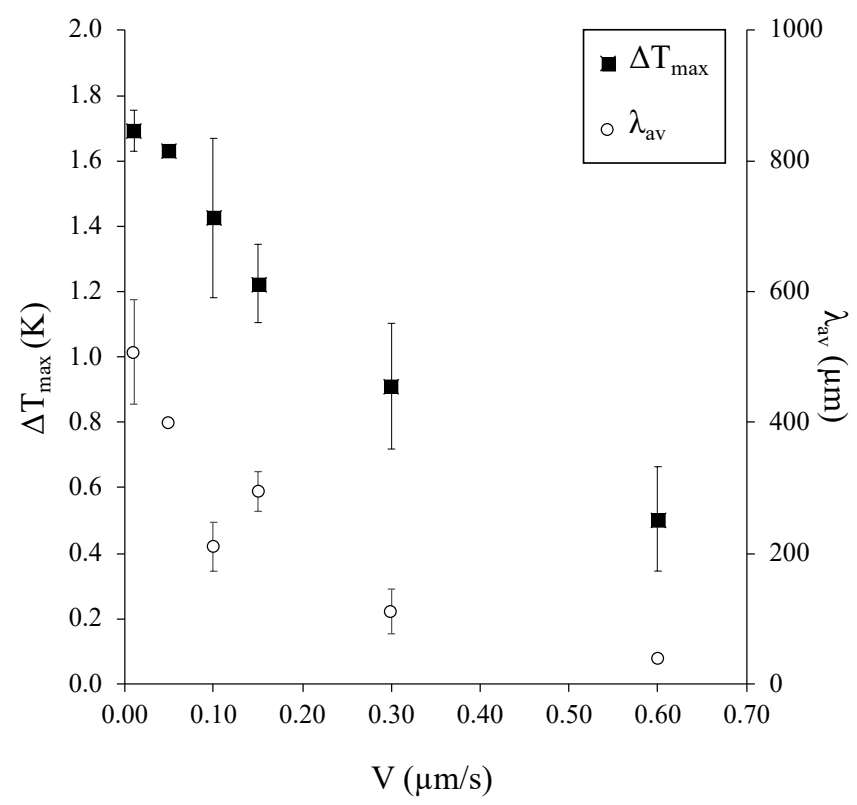

Fig. 2. Maximum temperature difference $\Delta \mathrm{T}_{\max }$ between an AMPD tip and the SCN-liquid interface, and average spacing $\lambda_{\text {av }}$ as a function of the pulling velocity $\mathrm{V}$. The error bars represent the experimental dispersion.

Noncrystallographic branching has been fairly extensively documented in spherulitic systems $[15,16]$. It designates basically the growth of a new crystal from a pre-existing one with a small misorientation (of a few degrees), without epitaxy or twinning [16]. Surprisingly, in the present system, noncrystallographic branching systematically, and apparently solely occurred at, or in the close vicinity of trijunctions [Fig. 1(c)]. It was therefore not associated with large undercoolings, but rather with atomic-scale instabilities at a trijunction line involving faceted interfaces. We did not identify any sign of twinning during this process. There was also no indication of a strong misorientation of the SCN crystal, but this remains to be more firmly attested. Crystallographic and nanoscale measurements that would be required for a deeper understanding of that process are currently out of reach for such organic compounds. 
Crystals of various orientations spanning a large angular range with respect to the parent crystal were generated by repeated noncrystallographic branching. Tilted AMPD crystals with a large inclination were eliminated. A dynamic balance was established over long times between noncrystallographic branching and crystal elimination. Hence, the number of AMPD crystals $\mathrm{N}_{\mathrm{A}}$ in an observation window (width $\mathrm{W}$ ) and the AMPD volume fraction in the solid remained statistically constant on average. We measured the average spacing $\lambda_{\mathrm{av}}=\mathrm{W} / \mathrm{N}_{\mathrm{A}}$ as a function of $\mathrm{V}$ (Fig. 2). As usual in eutectic growth, $\lambda_{\mathrm{av}}$ decreased when $\mathrm{V}$ increased. However, $\lambda_{\mathrm{av}}$ was unusually large, as compared to typical values in regular eutectics. We could not adjust a $\mathrm{KV}^{-1 / 2}$ curve on the $\lambda_{\mathrm{av}}(\mathrm{V})$ data with a reasonable value of the $\mathrm{K}$ constant. Moreover, if one assumes a value of the solute diffusion coefficient D on the order of 100 $\mu \mathrm{m}^{2} \mathrm{~s}^{-1}$, the ratio $\lambda_{\mathrm{av}} / \mathrm{l}_{\mathrm{d}}$ varied between 0.1 and $0.5\left(\mathrm{l}_{\mathrm{d}}=\mathrm{D} / \mathrm{V}\right.$ is the diffusion length), which is at least one order of magnitude larger than in regular eutectics.

For verification purposes, we also performed DS experiments in $100-\mu \mathrm{m}$ thick samples. Again, we observed decoupled microstructures at low V [Fig. 1(d)], and unsteady coupled ones at intermediate velocities [Fig. 1(e)]. The growth modes are therefore selected as a function of the growth velocity-(also see Ref. [9]), and are not specific to very thin samples.

Let us briefly describe the transient stages that followed downward or upward velocity jumps during solidification. Upon a downward $\mathrm{V}$ jump (typically from 0.6 to $0.1 \mu \mathrm{ms}^{-1}$ ), many AMPD crystals were eliminated and a fully decoupled growth regime was recovered. Locating a sharp threshold, if exists, would require more systematic experiments. An example of a transient microstructure after an upward velocity jump is shown in Fig. 3(a). Immediately after the velocity jump, the SCN-liquid interface was observed to recoil (as expected in a system with a small partition coefficient), and bend between two AMPD crystals, thus forming large liquid depressions with increasing undercooling. A rapid two-phased invasion with multiple noncrystallographic branching was observed [Fig. 3(b)]. We shall mention the striking similarity of our observations with those obtained by synchrotron x-ray radiography in a metallic f/nf eutectic alloy $\left(\mathrm{Ni}-\mathrm{Ni}_{3} \mathrm{Sn}_{4}\right)$ [17]. 

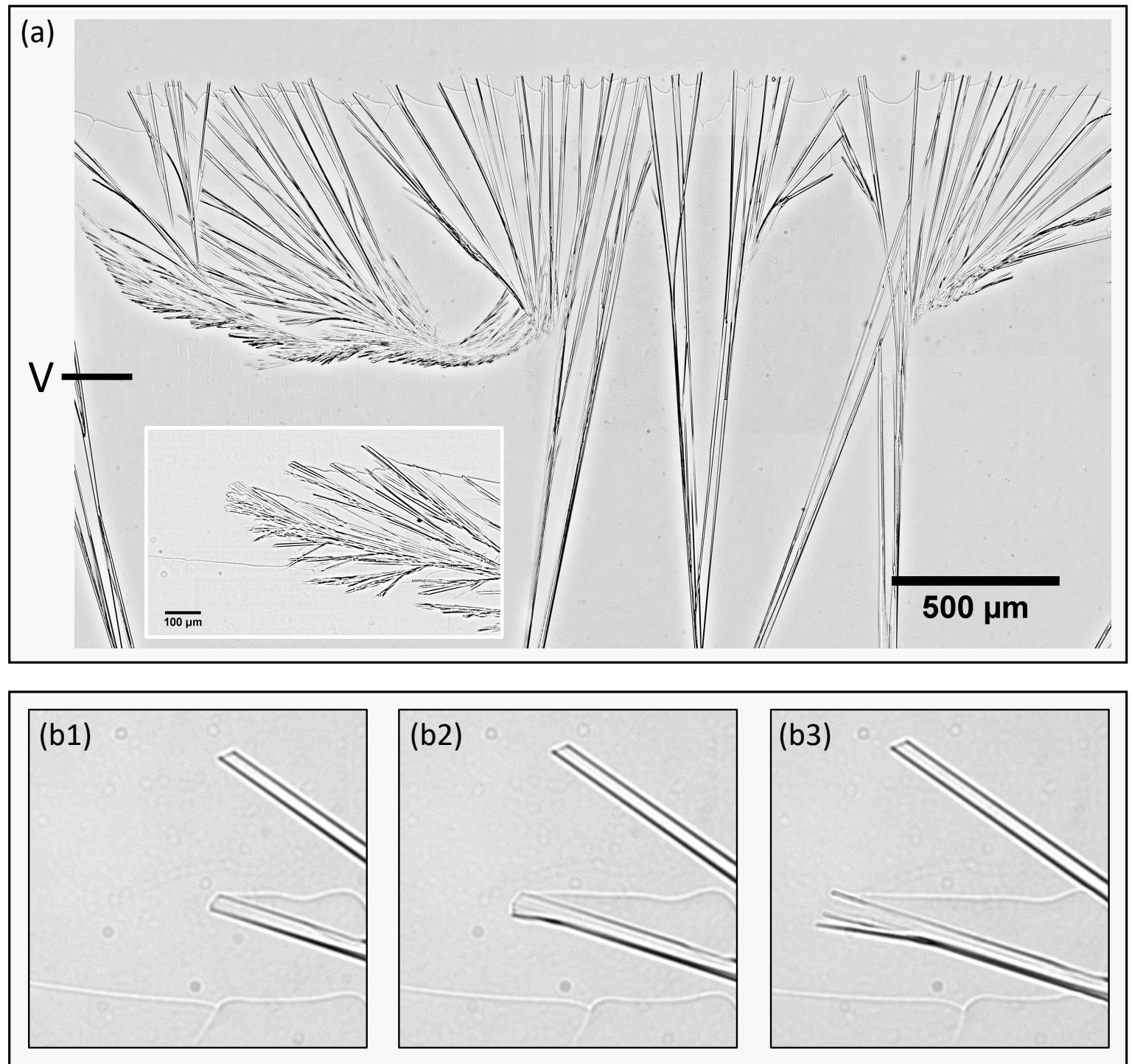

Fig. 3. Transient-growth morphologies obtained after an upward velocity jump during thin-sample directional solidification of an AMPD-SCN eutectic alloy. (a) Noncrystallographic branching and two-phased invasion events after a velocity jump from $0.3 \mu \mathrm{ms}^{-1}$ to $0.6 \mu \mathrm{ms}^{-1}$. The velocity change location is marked with "V" on the micrograph. Inset: two-phased invasion. (b) Time sequence showing of a noncrystallographic branching event (along with a decoupled AMPD crystal) after a velocity jump from 0.30 to $0.45 \mu \mathrm{ms}^{-1}$ (horizontal $^{\circ}$ dimension: $160 \mu \mathrm{m}$; time interval: $22 \mathrm{~s}$ ).

Two-phase fingers. For $\mathrm{V}$ higher than, say, $1 \mu \mathrm{ms}^{-1}$, the solidification dynamics was dominated by the formation of two-phase fingers (Fig. 4). A two-phase finger is a dendritelike growth shape made of a solid phase (here, $\mathrm{SCN}$ ) surrounding a thin crystal of another solid phase (AMPD) at the center, with a localized coupled growth at the tip. Two-phase fingers have been previously observed during univariant growth of a nonfaceted binary alloy in the presence of a small quantity of a third compound or a foreign impurity $[18,19]$-as in the present case. They are favored by unequal volume phase fractions in the eutectic solid. 
Here, long-lived two-phase fingers were such that the blocked AMPD facets were not exposed to the liquid but formed strongly anisotropic AMPD-SCN interphase boundaries in the solid, thus imposing the growth direction. Two-phase AMPD-SCN fingers [Fig. 4(b)] were unstable against two mechanisms: (i) a bending of the two-phase finger [Fig. 4(a)], which, in the present system, coincided with a plastic deformation of the AMPD crystal, followed by noncrystallographic branching; (ii) a depinning of the trijunction [Fig. 4(c)]. Structures locally resembling to two-phase fingers were also observed during transients [Fig. 3(b)].

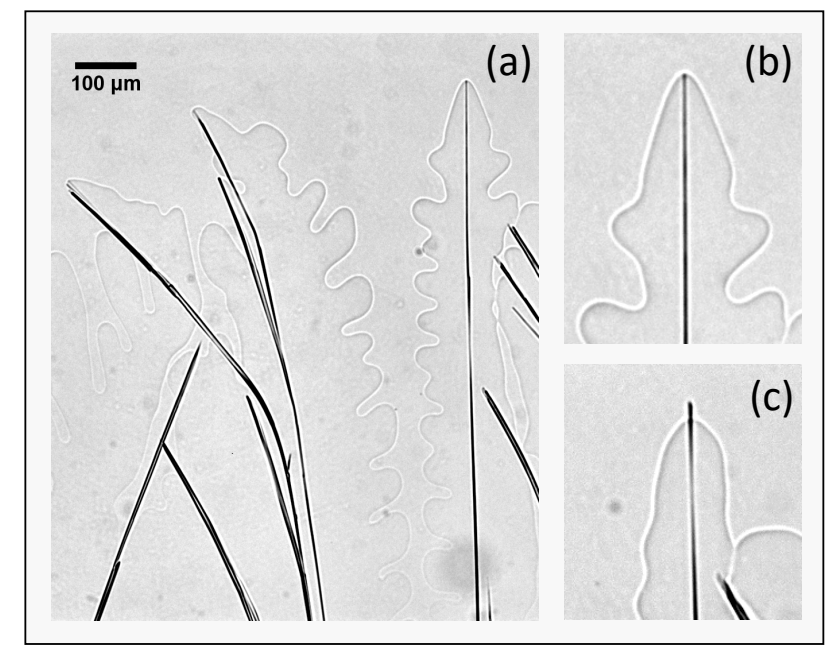

Fig. 4. (a) Asymmetric (on the left) and symmetric (on the right) faceted/nonfaceted two-phase fingers. AMPD and SCN are coupled at the tip of the two-phase finger in (b) and decoupled in (c). Image (b): large-magnification view of the symmetric two-phase finger shown in (a). Image (a) and (b) are taken at time $t_{1}$, and (c) at $t_{1}-723 \mathrm{~s}$. $\mathrm{V}=0.5 \mu \mathrm{ms}^{-1}$ [the horizontal dimension of images (a) and (b): $200 \mu \mathrm{m}$ ].

Conclusion. Real-time observations during directional solidification of a slightly hypoeutectic transparent faceted/nonfaceted eutectic alloy (AMPD-SCN) allowed us to identify (i) the existence of a quasi steady-state decoupled-growth regime with crystal needles growing ahead of a nonfaceted matrix; (ii) the occurrence of noncrystallographic branching at trijunctions; (iii) a transition from decoupled to coupled growth as a function of the solidification velocity, based on interfacial-kinetics effects; (iv) the formation of twophase fingers in a faceted/nonfaceted eutectic system. During decoupled growth, the faceted phase grows at a higher temperature than the nonfaceted one -similarly to the $\mathrm{Ni}_{3} \mathrm{Sn}_{4}$ needles in Ref. [17] . Trijunctions involving faceted interfaces were poorly stable, and prone to frequent depinning. The occurrence of noncrystallographic branching at the trijunctions was an unexpected phenomenon. Real-time observations during steady-state and transient regimes in a low-melting, transparent alloy were both key to our findings, and strikingly resembling 
previous observations in metallic systems. The thus evidenced features of the decoupled and unsteady coupled-growth regimes enter into contradistinction to oversimplified assumptions underlying previous theoretical work on irregular eutectics. The observation of faceted/nonfaceted two-phase fingers at higher growth velocities may also explain microstructural changes in multicomponent industrial alloys, such as Al-Si based alloys, leadfree solders, and cast iron. We hope that this study will inspire further theoretical and numerical-simulation developments.

\section{References}

[1] W. Kurz and D. J. Fisher, Fundamentals of Solidification, 1979.

[2] J. A. Dantzig and M. Rappaz, Solidification Second ed., EPFL Press 2017.

[3] A. Hellawell, Progress in Materials Science, 15 (1970) 3-78.

[4] K. A. Jackson and J. D. Hunt, Transactions of the Metallurgical Society of AIME, 236 (1966) 1129.

[5] D. J. Fisher and W. Kurz, Acta Metallurgica, 28 (1980) 777-794.

[6] E. Guzik and D. Kopyciński, Metallurgical and Materials Transactions A, 37 (2006) 3057-3067.

[7] A. J. Shahani, X. Xiao, and P. W. Voorhees, Nature Communications, 7 (2016) 12953.

[8] S. C. Huang and M. E. Glicksman, Acta Metallurgica, 29 (1981) 701-715.

[9] V. T. Witusiewicz, L. Sturz, U. Hecht, and S. Rex, Acta Materialia, 53 (2005) 173-183.

[10] T. Börzsönyi, S. Akamatsu, and G. Faivre, Physical Review E, 80 (2009) 051601.

[11] S. Akamatsu, S. Bottin-Rousseau, M. Serefoglu, and G. Faivre, Acta Mater., 60 (2012) 3199-3205.

[12] U. de la Torre, J. Lacaze, and J. Sertucha, International Journal of Materials Research, 107 (2016) 1041-1050.

[13] D. A. Pawlak, S. Turczynski, M. Gajc, K. Kolodziejak, R. Diduszko, K. Rozniatowski, J. Smalc, and I. Vendik, Advanced Functional Materials, 20 (2010) 1116-1124.

[14] M. Kaczkan, D. A. Pawlak, S. Turczyński, and M. Malinowski, Optical Materials, 33 (2011) 1519-1524.

[15] H. D. Keith and F. J. Padden, Journal of Applied Physics, 34 (1963) 2409-2421.

[16] J. Bisault, G. Ryschenkow, and G. Faivre, Journal of Crystal Growth, 110 (1991) 889909.

[17] N. Hou, S. A. Belyakov, L. Pay, A. Sugiyama, H. Yasuda, and C. M. Gourlay, Acta Materialia, 149 (2018) 119-131. 
[18] S. Akamatsu, G. Faivre, and T. Ihle, Physical Review E, 51 (1995) 4751-4773.

[19] S. Akamatsu and G. Faivre, Physical Review E, 61 (2000) 3757-3770. 